\title{
Effect of corticosteroids on wound healing after endoscopic sinus surgery*
}

\author{
M. Jorissen ${ }^{1}$ C. Bachert ${ }^{2}$ \\ ENT Department, HNS, University of Leuven, Leuven, Belgium \\ Department of Oto-Rhino-Laryngology, University Hospital Ghent, Ghent, Belgium
}

\begin{abstract}
SUMMARY
Background: Approximately 20\% patients with chronic rhinosinusitis undergoing Functional Endoscopic Sinus Surgery (FESS) experience impaired wound healing, leading to recurrences of sinusitis and polyps.

Methods: We investigated the efficacy of oral+ local steroids in wound healing, following FESS in subjects with a chronic rhinosinusitis with/without nasal polyps. Ninety-nine subjects were randomised to receive 6 months' treatment with mometasone furoate nasal spray (MFNS) $200 \mu \mathrm{g}$ bid or placebo in double-blind manner approximately 2 weeks after FESS. Postoperative mean total score for several endoscopic parameters scores assessed at 6 months was calculated as the primary efficacy end-point. An endoscopic combination score (for inflammation, oede$m a$, and polyps), a total symptoms score, and percent subjects requiring rescue medication, were assessed as secondary end-points.

Results: MFNS led to greater, although not significant, reductions in total endoscopic scores in all subjects, compared with placebo. The combination scores, however, indicated significantly improved healing with MFNS than with placebo for all subjects (median scores: 0.0 vs 2.0, $p=$ 0.02), and particularly for subjects with nasal polyps (median scores: 2.0 vs 4.0, $p=0.03$ ). The total symptom scores and percent subjects requiring rescue medication were similar in the two groups. MFNS was well tolerated.
\end{abstract}

Conclusions: These results suggest that treatment with MFNS following sinus surgery may improve wound healing, particularly in subjects with nasal polyps.

Key words: chronic rhinosinusitis, FESS, mometasone furoate, nasal polyps, wound healing

\section{INTRODUCTION}

Chronic rhinosinusitis without (CRSsNP) and with (CRSwNP) nasal polyps are frequently occurring diseases of the nasal and sinus mucosa, which respectively affect between $2-16 \%{ }^{(1,2)}$ and around $2-3 \%{ }^{(3,4)}$ of the population worldwide. Pathophysiological investigations have indicated that inflammation of the mucous membranes in the nasal cavity and paranasal sinuses often associated with bacterial infections, allergy/asthma, and mucociliary dysfunction is a prominent feature in both conditions ${ }^{(2,3)}$. However, one recent study has indicated that CRSsNP and CRSwNP can be distinguished as distinct disease entities, based on their inflammatory cell and mediator profiles (5). Recommendation of initial therapy includes topical corticosteroids and long-term antibiotics, followed by surgical intervention in patients not responding to pharmacotherapy ${ }^{(2,3,6)}$. Although Functional Endoscopic Sinus Surgery (FESS) is the standard surgical approach for both chronic rhinosinusitis and nasal polyps which have not responded to medical treatment, approximately $20 \%$ of patients experience impaired wound healing following FESS, leading to recurrences of sinusitis or complications requiring further surgery.

Wound healing is a highly ordered and well-coordinated process; involving proliferation of inflammatory cells to the wound, epithelial closure, cell differentiation, matrix deposition and remodelling; regulated by a wide variety of growth factors and cytokines ${ }^{(7)}$. Indeed, studies of subjects with chronic rhinosinusitis and nasal polyps undergoing FESS have demonstrated that the expression of inflammatory-cell derived matrix metalloproteinase (MMP)-9 is increased in the extracellular matrix during wound healing and is linked to poor healing quality ${ }^{(8)}$. After surgery, all patients require regular gentle suction cleaning for several months, irrigation and medical treatment with antibiotics and topical and systemic steroids to contain the inflammatory processes; which if left unchecked could possibly result in a vicious cycle that maintains chronic inflammation leading to recurrence of sinusitis and nasal polyposis ${ }^{(7)}$. It has been suggested that appropriate sustained post- 
surgical medical therapy is likely to benefit a significant proportion of patients who do not achieve long-term relief from surgery for chronic rhinosinusitis and nasal polyps ${ }^{(1,9)}$ and long-term therapy with intranasal steroids is therefore often included in postoperative treatment regimens for nasal polyposis to promote wound healing ${ }^{(10)}$. Indeed, postoperative steroid treatment regimen is supported by a recent study in New Zealand white rabbits, which investigated the effect of continuous topically released dexamethasone on surgical created $4 \mathrm{~mm}$ circular wounds on the medial side of the maxillary sinus in these animals ${ }^{(11)}$. The authors demonstrated that dexamethasone led to a significantly decreased extent of granulation and improved wound healing compared to control animals. Furthermore, dexamethasone significantly decreased the thickness of the stroma, without impeding epithelial differentiation. In contrast, treatment with short-term antibiotics has not been found to influence the immediate postoperative clinical evolution after endoscopic sinus surgery and antibiotics are generally not recommended for routine clinical use under these circumstances ${ }^{(12,13)}$.

However, studies of the efficacy and tolerability of various intranasal steroids in postoperative treatment have produced varying results. While an early study has demonstrated beclomethasone to significantly reduce the recurrence of nasal polyps, one year after simple polypectomy ${ }^{(14)}$, studies investigating the effects of fluticasone propionate have produced conflicting results. Although fluticasone propionate was not found to significantly reduce either symptoms or recurrence rates of chronic rhinosinusitis and nasal polyps in patients with nasal polyposis or chronic rhinosinusitis at 1 year after FESS ${ }^{(15)}$, a longer-term study in patients with CRSwNP demonstrated significant improvements in endoscopic polyp scores and total nasal volumes with fluticasone propionate than with placebo, 5 years after FESS ${ }^{(16)}$. More recently, one study investigated the effect of budesonide in uncontrolled case series of postoperative chronic sinusitis patients with or without nasal polyposis (17). The authors demonstrated that although patient and physician global assessments of disease were significantly improved, at 8-80 weeks (mean $=31.5$ weeks) after treatment with budesonide, there were no significant improvements in mucosal oedema, nasal discharge or nasal polyps, compared with pretreatment. There is some evidence that mometasone furoate may decrease the recurrence and relapse in nasal polyposis following endoscopic sinus surgery ${ }^{(18,19)}$, suggesting the possibility of mometasone furoate-mediated improvements in the wound healing process.

In view of these findings the objective of the present study was to investigate whether treatment with mometasone furoate nasal spray (MFNS) improves wound healing in subjects who have undergone FESS for bilateral nasal polyposis or chronic rhinosinusitis.

\section{MATERIALS AND METHODS \\ Patients}

Male and female patients (age $\geq 18$ years) suffering from bilateral nasal polyposis and/or chronic sinusitis, as diagnosed by history, nasal endoscopy and CT-scan were recruited into the study from two university hospital out-patient clinics in Belgium. All patients had failure to conventional medical treatment or contra-indications to medical treatment and required FESS for their disease.

Patients who had undergone sinus surgery in the last five years and those with surgical contra-indications, primary ciliary dyskinesia, asthma requiring inhalant corticosteroid treatment, aspirin hypersensitivity, immunodeficiency or cystic fibrosis were excluded from the study. Similarly, patients who had received systemic and topical corticosteroids within 4 weeks, intramuscular corticosteroids within 3 months, antihistamines or leukotriene receptor antagonists within 10 days, nasal decongestants within 24 hours, and nasal sodium cromolyn, atropine or ipratropium bromide, or antifungals within 1 week of screening for inclusion in the study were excluded. Patients with contra-indications for intranasal or oral use of corticosteroids or hypersensitivity to the study drugs were also excluded as were pregnant or breast feeding women.

\section{Study design}

This was a 2-arm, randomized, double-blind, placebo-controlled, prospective study. The study involved a total of eight visits, of which at visit 1 (screening visit) all subjects provided written informed consent and were evaluated for medical history and symptoms of disease. All subjects underwent nasal endoscopic examination and eligible individuals were scheduled for FESS at visit 2. At visit 1, the subjects were also randomised to receive either i) orally administered betamethasone $2 \mathrm{mg}$ tablets (Celestone ${ }^{\circledR}$ ) for seven days, followed by topically administered MFNS $200 \mu \mathrm{g}$ b.i.d for six months, or ii) matched placebo tablets and intranasal spray for seven days and six months, respectively, after FESS. Randomization to treatment was achieved according to a computer-generated sequential list, which was provided to each participating centre's pharmacy for distribution of appropriate study medication to the investigator and subsequently to the patient, in a double-blinded manner. Betamethasone matching placebo tablets were prepared by the pharmacy at University Hospital Gent, whereas MFNS (Nasonex ${ }^{\circledR}$ ) and matching placebo nasal sprays were provided by Schering-Plough. Following FESS, the subjects returned to the clinic after seven days (visit 3), 14 days (visit 4), 1 month (visit 5), 2 months (visit 6), 4 months (visit 7), and 6 months (visit 8); during which they underwent nasal examinations by endoscopy and evaluation of compliance with treatment, adverse events (AEs) and concomitant medications according to diary card records. FESS involved the stepwise approach to open the diseased sinuses and remove visibly altered mucosa; starting with the resection of the uncinate process, followed by the opening of the maxillary sinus, the 
bulla ethmoidalis, the ethmoidal cells, and, if necessary, the frontal and sphenoid sinuses. The middle turbinate was spared whenever possible.

During the study period, additional visits to the physician were allowed without restriction and the subjects were allowed to receive unrestricted nasal irrigation (without steroids) by whatever technique, at the discretion of the investigator. In case of an infection of the nasal cavities with persistent purulent secretions and/or pain that persisted for longer than 3 days, rescue medication comprising of a 7-10 day course of oral nonsteroidal anti-inflammatory drugs (NSAID) in combination with an antibiotic treatment (Augmentin 4x500 mg) was advocated up to a maximum of two courses during the study period at the discretion of the investigator. The additional physician visits and rescue medication were recorded in the CRF.

The study was approved by the local Ethical Committees or Review Boards of the participating centres and carried out in accordance with the principles of Declaration of Helsinki and the International Conference on Harmonization ( $\mathrm{ICH}$ ) notes for Guidance on Good Clinical Practice.

\section{Efficacy assessments}

The primary end point was the investigator's assessment of treatment outcome, based on reduction in total scores for a panel of eight endoscopically evaluable nasal characteristics at the end of 6-months' treatment (or time of early termination). Each characteristic was examined in the sinus cavities in both nostrils at visits 2 to 8 , and scored on 3-point scales as shown:

i. Stenosis of the implemented sinus ostia: $0=$ no stenosis; $1=$ partial stenosis $; 2=$ total stenosis.

ii. Hyperplasia/polypoid changes: $0=$ no hyperplasia; $1=$ hyperplasia; $2=$ polypoid changes .

iii. Synechia: $0=$ no adhesions; $1=$ few adhesions; $2=$ extensive adhesions.

iv. Remucosalized areas: $0=$ total remucosalization; $1=$ partial remucosalization; 2 = no remucosalization .

v. Secretions: $0=$ absent; $1=$ clear secretions;

2 = mucopurulent secretions.

vi. Inflammation: $0=$ absent; $1=$ mild inflammation;

$2=$ severe inflammation

vii. Oedematous swelling: $0=$ absent; $1=$ mild; $2=$ severe. viii. Blood crusts: $0=$ absent; $1=$ mild; $2=$ severe.

Post-hoc combination score for inflammation, oedema, and polyps; postoperative total symptoms score; and the percentage of patients requiring rescue medication were assessed as secondary end points. The total symptoms score was assessed as a composite score for nasal blockage, nasal discharge, headache/facial pain/pressure, olfactory disturbance, and overall discomfort at months $1,2,4$ and 6 ; using a 0 to 10 points visual analogue scale ${ }^{(20)}$. In addition, the patient's opinion of treatment success was assessed similarly.

\section{Safety assessment}

Safety was assessed according to the adverse events (AEs) recorded throughout the 6-month treatment period.

\section{Statistical analyses}

Analyses of efficacy assessments were performed on the intent-to-treat (ITT) population, which was defined as all patients who underwent surgery and were given subsequent treatment. As there are no studies documenting the use of a validated scoring system to assess the postoperative endoscopic evolution of the nasal cavity and sinuses after FESS, it was not possible to perform any power calculations to estimate the sample size. However, as some of the evaluated parameters occur infrequently (e.g. ostium stenosis) and the healing process was evaluated after FESS for 2 different pathologies, assessment of 50 patients in each treatment arm was considered to be adequate for a pilot cohort study with the specified objectives.

Continuous variables were presented by the number of nonmissing items and mean and standard deviation, unless otherwise indicated, and the significance of differences between the treatment groups was assessed by means of a two-sample t-test for normally distributed variables and by Wilcoxon rank sum test otherwise. Categorical variables were presented by their observed frequencies and percentages relative to the number of non-missing items, and the significance of any differences between the treatment groups was assessed by the chi-square test. A linear mixed effects model, including factors for randomised treatment group and time and their interaction, was used to assess the evolution of the total endoscopic score throughout the study. The model also included a random intercept and slope to model correlations between the time points. Actual time since surgery was included as a continuous variable using a Nadaraya-Watson kernel regression ${ }^{(21)}$. Differences between the two treatments at Week 1, Week 2, Months 1, 24 and 6 were assessed and significance levels adjusted using Holmes'method to control the associated Type I error. The overall difference between treatments in the evolution of the scores over time was assessed using a likelihood ratio test.

All tests were 2-sided and assessed at the 5\% significance level. Statistical analyses were performed using SAS v8.2 and the statistical package $\mathrm{R} v$ 2.6.2.

\section{RESULTS}

\section{Subject disposition and demographics}

A total of 99 patients were randomized to treatment with MFNS ( $n=49)$ or placebo $(n=50)$, of whom $2 / 49$ and $4 / 50$ patients in the MFNS and placebo group, respectively, did not return to the site for surgery. A further 2 patients ( 1 in each group) did not start their randomised treatment after surgery. Therefore, the ITT population comprised 91 patients (46 (93.9\%) in the MFNS group and $45(90.0 \%)$ in the placebo group). Overall, 67 patients (35 (76.1\%) in the MFNS group 
and $32(71.1 \%)$ in the placebo group) completed the study. The group of 24 patients not completing the study comprised 12 patients who were lost to follow-up (6 patients each in the MFNS and placebo groups), 4 patients who withdrew due to protocol violations for rescue medication or non-compliance with treatment (2 patient each in MFNS and placebo groups), and 8 patients who withdrew for other reasons ( 3 patients in MFNS group and 5 in placebo group).

The demographic and baseline clinical characteristics of the patients demonstrated that both treatment groups were similar with respect to demographics and preoperative symptom scores, although there were significant differences with respect to the initial diagnoses of polyposis nasi and chronic sinusitis $(\mathrm{p}=0.046)$ (Table 1).

Table 1. Demographic and baseline clinical characteristics of patients comprising the ITT population.

\begin{tabular}{|c|c|c|c|}
\hline $\begin{array}{l}\text { Demographic/clinical } \\
\text { Characteristic }\end{array}$ & $\begin{array}{l}\text { MFNS } \\
(\mathrm{N}=46)\end{array}$ & $\begin{array}{l}\text { Placebo } \\
(\mathrm{N}=45)\end{array}$ & $\begin{array}{l}\text { Overall } \\
(\mathrm{N}=91)\end{array}$ \\
\hline \multicolumn{4}{|l|}{ Age (years) } \\
\hline Mean $( \pm \mathrm{SD})$ & $46.7 \pm 11.77$ & $48.2 \pm 13.30$ & $47.4 \pm 12.50$ \\
\hline \multicolumn{4}{|l|}{ Gender } \\
\hline Male (N (\%)) & $30(65.22 \%)$ & $29(64.44 \%)$ & $59(64.84 \%)$ \\
\hline Female (N (\%)) & $16(34.78 \%)$ & $16(35.56 \%)$ & $32(35.16 \%)$ \\
\hline \multicolumn{4}{|l|}{$\begin{array}{l}\text { Preoperative total } \\
\text { symptom score }\end{array}$} \\
\hline $\mathrm{N}$ & 45 & 41 & 86 \\
\hline Mean $( \pm \mathrm{SD})$ & $28.5 \pm 9.19$ & $28.6 \pm 8.25$ & $28.5 \pm 8.7$ \\
\hline \multicolumn{4}{|l|}{ Initial diagnosis } \\
\hline Polyposis nasi (N (\%)) & $16(34.78 \%)$ & $25(55.56 \%)$ & $41(45.05 \%)$ \\
\hline Chronic sinusitis (N (\%)) & $30(65.22 \%)$ & $20(44.44 \%)$ & $50(54.95 \%)$ \\
\hline
\end{tabular}

\section{Efficacy assessments}

Investigator's assessment of treatment outcome at end of treatment Table 2 shows the effect of treatment on total endoscopic scores after 6 months treatment.

The total endoscopic scores; assessed either for the group as a whole or according to initial diagnosis of disease; were lower in the MNFS-treated subjects than in the placebo-treated subjects, however, any differences between the treatments groups were not statistically significant.

Table 2. Total endoscopic scores (Investigator's assessment of outcome) at end of 6 months' treatment, assessed by group and by initial diagnosis.

\begin{tabular}{lccc}
\hline Treatment outcome & MFNS & Placebo & p-value \\
\hline Overall & & & \\
$\mathrm{N}$ & 46 & 45 & \\
Median (Q1; Q3) & $3.0(1.0 ; 7.0)$ & $5.0(1.0 ; 8.0)$ & 0.350 \\
\hline Polyposis Nasi & & & \\
$\mathrm{N}$ & 16 & 25 & \\
Median (Q1; Q3) & $2.5(1.0 ; 8.5)$ & $5.0(2.0 ; 12.0)$ & 0.340 \\
\hline Chronic Sinusitis & & & \\
$\mathrm{N}$ & 30 & 20 & \\
Median (Q1; Q3) & $3.0(1.0 ; 7.0)$ & $4.5(0.5 ; 8.0)$ & 0.905 \\
\hline
\end{tabular}

Assessment of the evolution of total endoscopic scores over the course of treatment demonstrated that these were progressively decreased over time in both treatment groups. Differences in the evolution of the scores between the treatment groups were not statistically significant $(p=0.391)$. The treatment effect was also not significantly different, when assessed according to the initial diagnosis $(\mathrm{p}=0.909)$.

Combination endoscopic scores for inflammation, oedema, and polyps scores

A post-hoc analysis of the combination score for the clinically relevant features of inflammation, oedema, and polyps at 6 months showed that this was significantly improved in MFNStreated subjects, compared with placebo-treated subjects overall, and specifically for subjects with nasal polyps, but not for subjects with chronic sinusitis (Table 3).

Table 3. Combination endoscopic scores for inflammation, oedema, and polyps scores at end of 6 months' treatment.

\begin{tabular}{lccc}
\hline Treatment outcome & MFNS & Placebo & p-value \\
\hline Overall & 44 & 43 & \\
N & & & \\
Median (Q1; Q3) & $0.0(0.0 ; 2.0)$ & $3.0(0.0 ; 6.0)$ & 0.02 \\
\hline Polyposis Nasi & 16 & 24 & \\
N & & & 9 \\
Median (Q1; Q3) & $2.0(0.0 ; 3.0)$ & $4.0(0.0 ; 6.0)$ & 0.02 \\
\hline Chronic Sinusitis & & & \\
N & 28 & 19 & \\
Median (Q1; Q3) & $0.0(0.0 ; 2.0)$ & $2.0(0.0 ; 4.0)$ & 0.694 \\
\hline
\end{tabular}

Assessment of the evolution of combination scores over the course of treatment demonstrated that overall MFNS was significantly more effective than placebo $(p=0.017)$ (Table 4). However, after adjusting the analysis for the initial diagnosis, this effect was no longer found to be statistically significant ( $p$ $=0.142)$ (Figure 1).

Post-operative total symptoms score

The results for the total symptom scores at the last visit and the associated change from baseline indicated that although

Table 4. Evolution of combination endoscopic scores for inflammation, oedema, and polyps over a course of 6 months' treatment.

\begin{tabular}{lccc}
\hline Treatment period & $\begin{array}{c}\text { MFNS } \\
(\text { mean } \pm \text { SEM) }\end{array}$ & $\begin{array}{c}\text { Placebo } \\
(\text { mean } \pm \text { SEM) }\end{array}$ & p-value \\
\hline Week 1 & $3.73 \pm 0.23$ & $3.99 \pm 0.23$ & 0.011 \\
\hline Week 2 & $3.28 \pm 0.22$ & $3.84 \pm 0.22$ & 0.011 \\
\hline Month 1 & $2.50 \pm 0.26$ & $3.56 \pm 0.26$ & 0.011 \\
\hline Month 2 & $1.49 \pm 0.35$ & $3.00 \pm 0.35$ & 0.011 \\
\hline Month 4 & $1.62 \pm 0.40$ & $2.25 \pm 0.40$ & 0.246 \\
\hline Month 6 & $1.55 \pm 0.44$ & $2.75 \pm 0.43$ & 0.091 \\
\hline
\end{tabular}

$\mathrm{SEM}=$ standard error.

Estimates were obtained using a linear mixed model including time since FESS, treatment and their interaction as fixed effects. Significance levels were adjusted using Holmes' method. 


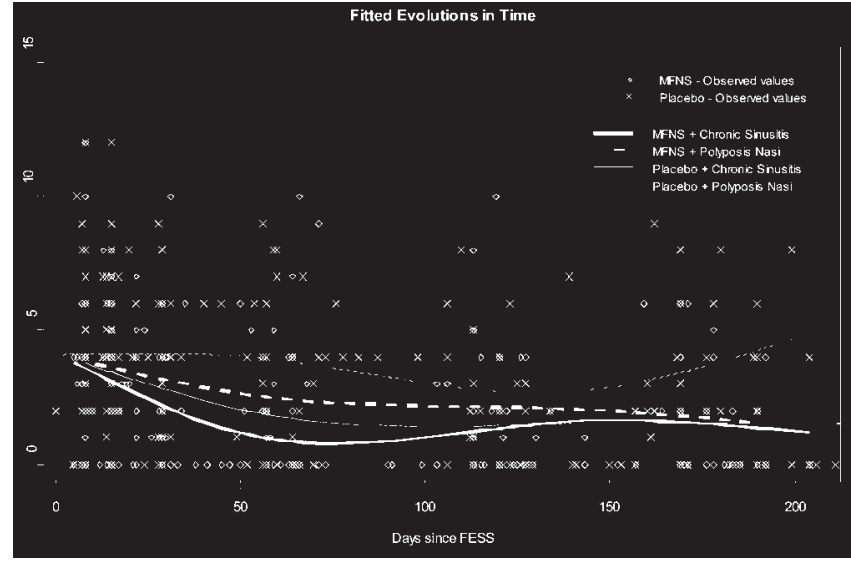

Figure 1. Evolution of combination scores in patients with CRSsNP and CRSwNP, treated with MFNS (Nasonex ${ }^{\circledR}$ ) or placebo.

MFNS decreased the symptom scores from baseline, this effect was not significantly different from that observed for placebo $(14.3 \pm 9.10$ for MFNS vs $13.8 \pm 8.63$ for placebo; $p=0.908)$.

\section{Rescue medication}

The proportion of subjects requiring rescue medication in the MFNS-treated group (9/46 (19.6\%) was not significantly different from the proportion of subjects requiring rescue medication in the placebo-treated (7/45 (15.6\%); $\mathrm{p}=0.615)$.

\section{Safety}

Overall, $63.0 \%$ of subjects in the MFNS and $62.2 \%$ of subjects in placebo group experienced at least one adverse event (AE). The most common AEs experienced by $\geq 5 \%$ of patients in MFNS and placebo groups were headache $(28.3 \%: 17.8 \%$ of subjects, respectively), sinusitis (4.3\%: $11.1 \%$ of subjects, respectively), paranasal sinus infection (6.5\%:6.7\% of subjects respectively), and cold (4.3\%: $6.7 \%$ of subjects, respectively). Serious AEs were rare, and occurred in 2 (4.3\%) and 3 (6.7\%) subjects in the MFNS and placebo group, respectively. Similarly, drug-related AEs were few and occurred in 5 (10.9 $\% ; 2$ with acute sinusitis and 1 each with headache, epistaxis and rhinorrhoea) and 1 (2.2\%; dizziness) subject in the MFNS and placebo group, respectively.

\section{DISCUSSION}

There is little doubt that the efficiency of wound healing in the nasal passages following endoscopic sinus surgery for CRSwNP or CRSsNP is likely to influence the recurrence rates for nasal polyposis or sinusitis, and/or complications requiring further surgery. In the absence of a validated monitoring system to assess the evolution of postoperative wound healing processes in the nasal cavity and sinuses after FESS, and the relatively little information available on the efficacy of specific therapies in promoting these wound healing processes, this pilot study was specifically designed to investigate the effect of mometasone furoate nasal spray on a variety of endoscopically evaluable nasal features, which reflect wound healing. The study demonstrated that although the total endoscopic score for the different nasal features was progressively decreased from baseline to a greater extent over the course of the study in MFNS-treated patients than in placebo-treated patients, the difference in the decrease in the scores between the two groups was not significant. However, assessment of wound healing based on a combination score for the more clinically relevant features of inflammation, oedema, and polyps indicated that treatment with MFNS following FESS was significantly more effective than treatment with placebo in improving wound healing. Furthermore, subjects with an initial diagnosis of nasal polyps (CRSwNP) were more likely to experience MFNS-mediated improvements in wound healing than subjects with an initial diagnosis of chronic rhinosinusitis without polyps (CRSsNP). Assessment of the postoperative total symptoms score indicated that although this was decreased from preoperative baseline values in both treatment groups, the effect for MNFS was not significant compared with placebo. Overall, MFNS was well tolerated over the 6-month treatment period, as indicated by similarities with placebo for the percent of subjects discontinuing the study, the need for rescue medications, and the incidence and types of AEs experienced.

While the efficacy and safety of MFNS in the prophylactic treatment of allergic rhinitis is well established ${ }^{(22)}$, an increasing body of evidence suggests that MFNS may be effective also in the treatment of rhinosinusitis and nasal polyposis. Early studies in patients with acute rhinosinusitis demonstrated that MFNS $200 \gamma$ b.i.d used as adjunct therapy to antibiotics led to significantly greater symptom relief than adjunct placebo (i.e. antibiotic therapy alone) ${ }^{(23,24)}$. Indeed, a direct comparison between MFNS and amoxicillin or placebo in patients with acute, uncomplicated rhinosinusitis demonstrated that MFNS was significantly superior to both amoxicillin and placebo in improving overall and most individual symptom scores, as well as providing a significantly greater global response to treatment (25). More recently, one randomised controlled trial compared the effect of MFNS $200 \gamma$ once- or twice-daily, amoxicillin $500 \mathrm{mg}$ t.i.d, and placebo on the health-related quality of life (HRQoL) of patients with acute, uncomplicated rhinosinusitis, and demonstrated that only MFNS b.i.d. significantly improved the overall HRQoL scores in these individuals, compared with placebo ${ }^{(26)}$. Similarly, well-controlled trials of MFNS $200 \gamma$ once- or twice-daily in patients with bilateral nasal polyps have demonstrated that MFNS was significantly superior to placebo in reducing polyp grade (size and extent), and improving the symptoms of nasal congestion and/or obstruction, sense of smell, peak nasal inspiratory flow, and QoL measures ${ }^{(27-30)}$. Moreover, a beneficial and lasting improvement in the symptoms of nasal polyposis was noted within 24 hours to 5 days of initiating therapy with MFNS ${ }^{(28)}$. 
Although these studies investigated the effect of MFNS therapy in patients with mild to moderate disease not requiring surgery, some studies have suggested that postoperative mometasone furoate may also be beneficial in the management of patients who have undergone surgery for nasal polyposis ${ }^{(17,18)}$. A study comparing the effects of furosemide, mometasone furoate and placebo on postoperative relapse of chronic hyperplastic sinusitis with nasal polyposis demonstrated that patients treated with furosemide or mometasone experienced predominantly early stage nasal polyposis relapse, where as patients treated with placebo experienced significantly more severe grades of nasal polyposis relapse; as classified according to a pre-determined grading system using fibre-optic nasal endoscopy prior to endoscopic surgery ${ }^{(18)}$. Moreover, both active treatments maintained nasal patency, measured by acoustic rhinometry, in the physiological range during the first 3 years after surgery, whereas the nasal volumes progressively worsened in the placebo-treated patients over this period. Similarly, preliminary findings of a study investigating the effect of MFNS in patients undergoing FESS for bilateral nasal polyps have indicated that MNFS was significantly superior to placebo in prolonging the time to recurrence in these subjects ${ }^{(19)}$. Collectively, these findings for the postoperative effects of mometasone furoate suggest that this agent may aid in woundhealing following endoscopic surgery for chronic sinusitis and nasal polyposis.

To our knowledge this is the first study to assess wound healing following FESS by thorough monitoring of a variety of parameters, which are likely to be involved in the wound healing process. In the absence of comparative studies and relevant standardised procedure/s for evaluation of wound-healing following nasal surgery, our results suggest that the retrospectively chosen combination score for the presence of postoperative inflammation, oedema, and polyps could be a useful tool for generally evaluating postoperative wound-healing and the effect of therapy on this process, following FESS for chronic rhinosinusitis and nasal polyposis. This is especially so because this combination score comprises individual scores for components that are prominent and clinically relevant features in the pathophysiology of these diseases, while at the same time it excludes scores for uncommon and quantitatively less important endoscopic findings, such as stenosis and bleeding in the short term for example. In view of the established efficacy of MFNS in the attenuation of the symptoms of acute rhinosinusitis ${ }^{(23-25)}$ and nasal polyposis patients ${ }^{(27-30)}$, a slightly surprising finding of this pilot study was the lack of significance in the difference between the postoperative mean total symptoms scores noted for MFNS- and placebo-treated subjects. This may be a consequence of several parameters, of which the sample size is likely to be important. It was not possible to power this study initially due to the lack of comparative data. However, using data from the present study, retrospective sample size calculations were performed based on the use of the
Wilcoxon rank-sum test to assess differences between the groups. Calculations yielded a probability of 0.52 in the overall population for a patient in the MFNS group having a significantly lower value for the primary endpoint than a patient in the placebo group, whereas in the nasal polyps group this probability was calculated to be 0.56 . Therefore, a sample size of 5110 patients in each treatment group for the overall population and 364 patients in each treatment group for the nasal polyps group would be required to obtain a statistically significant treatment effect with a power of $80 \%$, using a $5 \%$ twosided significance level. It is also possible that the lack of significance in the mean total symptom scores noted for the MFNS- and placebo-treated patients was i) a consequence of a highly successful surgical intervention itself, which attenuated the symptoms to a minimal level in both treatment groups, or ii) that recurrence of more severe/troublesome symptoms, which could demonstrably be attenuated to a significantly greater degree with MFNS than with placebo, may occur with recurrence of higher grade sinusitis and polyps over a longer than the six-month treatment period investigated in the present study. Indeed, Passàli and colleagues ${ }^{(18)}$ have demonstrated that the postoperative nasal patency in patients with chronic hyperplastic sinusitis with nasal polyposis was not significantly altered by treatment with furosemide, mometasone furoate, or placebo for up to 1-year post-endoscopic nasal surgery, and that significant active treatment effects became apparent over a much longer period of 3-6 years after surgery. Importantly, these authors additionally demonstrated that long-term treatment with neither furosemide- nor mometasone led to any significant adverse events in these patients. Indeed, a review on the safety of MFNS has recently indicated that long-term use of MFNS 100-200 $\gamma$ b.i.d. was safe and well tolerated; with no significant differences in topical or systemic adverse effects compared with placebo ${ }^{(31)}$. Moreover, an early multicenter study investigating the incidence of morphological changes in the nasal mucosa of patients with perennial rhinitis following treatment for 1 year with MFNS $200 \gamma$ b.i.d has demonstrated that this agent did not lead to any significant adverse tissue changes, including increase in epithelial thickness or tissue atrophy, although the numbers of inflammatory cells in the epithelium and lamina propria were significantly decreased, compared to baseline ${ }^{(32)}$.

In summary, the findings of this pilot study provide useful pointers for further studies and procedures for investigating wound-healing in subjects with chronic rhinosinusitis and nasal polyposis following FESS. Studies investigating the use of combination scores for clinically and pathologically relevant parameters over a period of at least three years after FESS are likely to provide useful data, and may possibly lead to the development of novel treatment options and strategies. Presently, however, given the efficacy and tolerability of MFNS, this agent may be considered for inclusion in a therapeutic regimen for patients recovering from FESS. 


\section{ACKNOWLEDGEMENTS}

The study was supported by Schering-Plough Corp.

\section{REFERENCES}

1. Aukema AAC, Fokkens WJ. Chronic rhinosinusitis: management for optimal outcomes. Treat Respire Med 2004; 3: 97-105.

2. Scadding GK, Durham SR, Mirakian R, et al. BSACI guidelines for the management of rhinosinusitis and nasal polyposis. Clin Exp Allergy 2008; 38: 260-275.

3. Fokkens W, Lund V, Mullol J, et al. European Position Paper on Rhinosinusitis and Nasal Polyps group. European position paper on rhinosinusitis and nasal polyps 2007. Rhinol Suppl 2007; 20 (Suppl 1): 1-136.

4. Hopkins C, Loh C, Roberts D. Medical versus surgical interventions for nasal polyps. (Protocol) Cochrane Database of Systematic Reviews 2008, Issue 1. Art. No.: CD006991.

5. Van Zele T, Claeys S, Gevaert P, et al. Differentiation of chronic sinus diseases by measurement of inflammatory mediators. Allergy 2006; 61: 1280-1289.

6. Fokkens W, Lund V, Bachert C, et al. EAACI position paper on rhinosinusitis and nasal polyps executive summary. Allergy 2005; 60: 583-601.

7. Lazard DS, Prulière-Escabasse V, Papon JF, Escudier E, Coste A. Blessure et réparation épithéliale: une hypothèse physiopathologique de la polypose nasosinusienne. Presse Med. 2007; 36: 1104-1108.

8. Watelet JB, Demetter P, Claeys C, Van Cauwenberge P, Cuvelier C, Bachert C. Neutrophil-derived metalloproteinase- 9 predicts healing quality after sinus surgery. Laryngoscope 2005; 115: 56-61.

9. Vining EM. Evolution of medical management of chronic rhinosinusitis. Ann Otol Rhinol Laryngol Suppl 2006; 196: 54-60.

10. Stammberger H. Surgical treatment of nasal polyps: past, present, and future. Allergy 1999; 54 (Suppl 53): 7-11.

11. Beule AG, Scharf C, Biebler KE, et al. Effects of topically applied dexamethasone on mucosal wound healing using a drug-releasing stent. Laryngoscope 2008; 118: 2073-2077.

12. Annys E, Jorissen M. Short term effects of antibiotics (Zinnat) after endoscopic sinus surgery. Acta Oto-rhino-laryngologica Belg 2000; 54: 23-28.

13. Jorissen M. Postoperative care following endoscopic sinus surgery. Rhinology 2004; 42; 114-120.

14. Virolainen E, Puhakka H. The effect of intranasal beclomethasone dipropionate on the recurrence of nasal polyps after ethmoidectomy. Rhinology 1980; 18: 9-18.

15. Dijkstra MD, Ebbens FA, Poublon RM, Fokkens WJ. Fluticasone propionate aqueous nasal spray does not influence the recurrence rate of chronic rhinosinusitis and nasal polyps 1 year after functional endoscopic sinus surgery. Clin Exp Allergy 2004; 34: 13951400 .

16. Rowe-Jones JM, Medcalf M, Durham SR, Richards DH, Mackay IS. Functional endoscopic sinus surgery: 5 year follow up and results of a prospective, randomised, stratified, double-blind, placebo controlled study of postoperative fluticasone propionate aqueous nasal spray. Rhinology 2005; 43: 2-10.

17. Kanowitz SJ, Batra PS, Citardi MJ.Topical budesonide via mucosal atomization device in refractory postoperative chronic rhinosinusitis. Otolaryngol Head Neck Surg 2008; 139: 131-136.

18. Passàli D, Bernstein JM, Passali FM, Damiani V, Passàli GC, Bellussi L. Treatment of recurrent chronic hyperplastic sinusitis with nasal polyposis. Arch Otolaryngol Head Neck Surg 2003; 129: 656-659.

19. Stjärne P, Olsson P. Mometasone furoate prolongs post-FESS return of nasal polyps. Otolaryngol Head Neck Surg 2007; 137 (Suppl 1): P273 (abstract).
20. Lund VJ, Kennedy DW. Quantification for staging sinusitis. The Staging and Therapy Group. Ann Otol Rhinol Laryngol (Suppl) 1995; 167: 17-21.

21. Nadaraya, EA. On Estimating Regression. Theory of Probability and its Applications 1964; 9: 141-142.

22. van Drunen C, Meltzer EO, Bachert C, Bousquet J, Fokkens WJ. Nasal allergies and beyond: a clinical review of the pharmacology, efficacy, and safety of mometasone furoate. Allergy 2005; 60 (Suppl 80): 5-19.

23. Meltzer EO, Charous BL, Busse WW, Zinreich SJ, Lorber RR, Danzig MR. Added relief in the treatment of acute recurrent sinusitis with adjunctive mometasone furoate nasal spray. The Nasonex Sinusitis Group. J Allergy Clin Immunol 2000; 106: 630637.

24. Nayak AS, Settipane GA, Pedinoff A, et al. Effective dose range of mometasone furoate nasal spray in the treatment of acute rhinosinusitis. Ann Allergy Asthma Immunol 2002; 89: 271-278.

25. Meltzer EO, Bachert C, Staudinger H. Treating acute rhinosinusitis: comparing efficacy and safety of mometasone furoate nasal spray, amoxicillin, and placebo. J Allergy Clin Immunol 2005; 116: 1289-1295.

26. Bachert C, Meltzer EO. Effect of mometasone furoate nasal spray on quality of life of patients with acute rhinosinusitis. Rhinology 2007; 45: 190-196.

27. Small CB, Stryszak P, Danzig M, Damiano A. Onset of symptomatic effect of mometasone furoate nasal spray in the treatment of nasal polyposis. J Allergy Clin Immunol 2008; 121: 928-932.

28. Small CB, Hernandez J, Reyes A, et al. Efficacy and safety of mometasone furoate nasal spray in nasal polyposis. J Allergy Clin Immunol 2005; 116:1275-1281.

29. Stjärne P, Blomgren K, Cayé-Thomasen P, Salo S, Søderstrøm T. The efficacy and safety of once-daily mometasone furoate nasal spray in nasal polyposis: a randomized, double-blind, placebo-controlled study. Acta Otolaryngol 2006; 126: 606-612.

30. Stjärne P, Mösges R, Jorissen M, Passàli D, Bellussi L, Staudinger H, Danzig M. A randomized controlled trial of mometasone furoate nasal spray for the treatment of nasal polyposis. Arch Otolaryngol Head Neck Surg 2006; 132: 179-185.

31. Zitt M, Kosoglou T, Hubbell J. Mometasone furoate nasal spray: a review of safety and systemic effects. Drug Saf 2007; 30: 317-326.

32. Minshall E, Ghaffar O, Cameron L, et al. Assessment by nasal biopsy of long-term use of mometasone furoate aqueous nasal spray (Nasonex) in the treatment of perennial rhinitis. Otolaryngol Head Neck Surg 1998; 118: 648-654.

Professor Mark Jorissen M.D., Ph.D. ENT Department, HNS

UZ Leuven

Kapucijnenvoer 33

B-3000 Leuven

Belgium

Tel: +32-16-33-2342

Fax: +32-16-33-2335

E-mail: mark.jorissen@uzleuven.be 